\title{
SPACES OF $D_{L^{p}}$ TYPE AND A CONVOLUTION PRODUCT ASSOCIATED WITH THE SPHERICAL MEAN OPERATOR
}

\author{
M. DZIRI, M. JELASSI, AND L. T. RACHDI
}

Received 2 June 2004 and in revised form 23 November 2004

We define and study the spaces $\mu_{p}\left(\mathbb{R} \times \mathbb{R}^{n}\right), 1 \leq p \leq \infty$, that are of $D_{L^{p}}$ type. Using the harmonic analysis associated with the spherical mean operator, we give a new characterization of the dual space $\mu_{p}^{\prime}\left(\mathbb{R} \times \mathbb{R}^{n}\right)$ and describe its bounded subsets. Next, we define a convolution product in $\mu_{p}^{\prime}\left(\mathbb{R} \times \mathbb{R}^{n}\right) \times M_{r}\left(\mathbb{R} \times \mathbb{R}^{n}\right), 1 \leq r \leq p<\infty$, and prove some new results.

\section{Introduction}

The spherical mean operator $\mathscr{R}$ is defined, for a function $f$ on $\mathbb{R}^{n+1}$, even with respect to the first variable, by

$$
\mathscr{R}(f)(r, x)=\int_{S^{n}} f(r \eta, x+r \xi) d \sigma_{n}(\eta, \xi), \quad(r, x) \in \mathbb{R} \times \mathbb{R}^{n},
$$

where $S^{n}$ is the unit sphere $\left\{(\eta, \xi) \in \mathbb{R} \times \mathbb{R}^{n}: \eta^{2}+\|\xi\|^{2}=1\right\}$ in $\mathbb{R}^{n+1}$ and $\sigma_{n}$ is the surface measure on $S^{n}$ normalized to have total measure one.

This operator plays an important role and has many applications, for example, in image processing of so-called synthetic aperture radar (SAR) data (see $[7,8]$ ), or in the linearized inverse scattering problem in acoustics [6]. In [10], the authors associate to the operator $\mathscr{R}$ a Fourier transform and a convolution product and have established many results of harmonic analysis (inversion formula, Paley-Wiener and Plancherel theorems, etc.).

In [11], the authors define and study Weyl transforms related to the mean operator $\mathscr{R}$ and have proved that these operators are compact. The spaces $D_{L^{p}}, 1 \leq p \leq \infty$, have been studied by many authors $[1,2,4,5,12,13]$. In this work, we introduce the function spaces $\mu_{p}\left(\mathbb{R} \times \mathbb{R}^{n}\right), 1 \leq p \leq \infty$, similar to $D_{L^{p}}$, but replace the usual derivatives by the operator

$$
L=l+\sum_{j=1}^{n}\left(\frac{\partial}{\partial x_{j}}\right)^{2},
$$


where $l$ is the Bessel operator defined on $] 0,+\infty[$ by

$$
l=\left(\frac{\partial}{\partial r}\right)^{2}+\frac{n}{r} \frac{\partial}{\partial r}
$$

The main result of this paper gives a new characterization of the dual space $\mathcal{M}_{p}^{\prime}(\mathbb{R} \times$ $\left.\mathbb{R}^{n}\right)$ of the space $M_{p}\left(\mathbb{R} \times \mathbb{R}^{n}\right)$ and a description of its bounded subsets. More precisely, in Section 2, we recall some harmonic results related to a convolution product and the Fourier transform connected with the spherical mean operator, that we use in the following sections.

In the Section 3, we define the space $\mu_{p}\left(\mathbb{R} \times \mathbb{R}^{n}\right), 1 \leq p \leq \infty$, to be the space of measurable functions $f$ on $] 0,+\infty\left[\times \mathbb{R}^{n+1}\right.$ such that for all $k \in \mathbb{N}, L^{k} f$ belongs to the space $L^{p}(d v)$ (the space of functions of $p$ th power integrable on $\left[0,+\infty\left[\times \mathbb{R}^{n+1}\right.\right.$ with respect to the measure $r^{n} d r \otimes d x$ ). We give some properties of this space, in particular we prove that it is a Frechet space.

Section 4 is consecrated to the study of the dual space $M_{p}^{\prime}\left(\mathbb{R} \times \mathbb{R}^{n}\right)$. We give a nice description of the elements of this space and we characterize its bounded subsets.

In the last section, we define and study a convolution product in $M_{p}^{\prime}\left(\mathbb{R} \times \mathbb{R}^{n}\right) \times$ $M_{r}\left(\mathbb{R} \times \mathbb{R}^{n}\right), 1 \leq r \leq p<\infty$, where $M_{r}\left(\mathbb{R} \times \mathbb{R}^{n}\right)$ is the closure of the Schwartz space $S_{*}\left(\mathbb{R} \times \mathbb{R}^{n}\right)$ in $\mathcal{M}_{r}\left(\mathbb{R} \times \mathbb{R}^{n}\right)$.

\section{Spherical mean operator}

In this section, we define and recall some properties of the spherical mean operator. For more details see $[3,6,10,11]$. We denote by

(A) $\mathscr{E}_{*}\left(\mathbb{R} \times \mathbb{R}^{n}\right)$ the space of infinitely differentiable functions on $\mathbb{R} \times \mathbb{R}^{n}$, even with respect to the first variable,

(B) $S^{n}$ the unit sphere in $\mathbb{R} \times \mathbb{R}^{n}$,

$$
S^{n}=\left\{(\eta, \xi) \in \mathbb{R} \times \mathbb{R}^{n} ; \eta^{2}+\|\xi\|^{2}=1\right\}
$$

where for $\xi=\left(\xi_{1}, \ldots, \xi_{n}\right)$, we have $\|\xi\|^{2}=\xi_{1}^{2}+\cdots+\xi_{n}^{2}$,

(C) $d \sigma$ the normalized surface measure on $S^{n}$.

Definition 2.1. The spherical mean operator is defined on $\mathscr{E}_{*}\left(\mathbb{R} \times \mathbb{R}^{n}\right)$ by

$$
\forall(r, x) \in\left[0,+\infty\left[\times \mathbb{R}^{n}, \quad \mathscr{R} f(r, x)=\int_{S^{n}} f(r \eta, x+r \xi) d \sigma_{n}(\eta, \xi) .\right.\right.
$$

For $(\mu, \lambda) \in \mathbb{C} \times \mathbb{C}^{n}$, we put

$$
\forall(r, x) \in\left[0,+\infty\left[\times \mathbb{R}^{n}, \quad \varphi_{\mu, \lambda}(r, x)=\mathscr{R}\left(\cos (\mu \cdot) e^{-i\langle\lambda / \cdot\rangle}\right)(r, x) .\right.\right.
$$

We have

$$
\varphi_{\mu, \lambda}(r, x)=j_{(n-1) / 2}\left(r \sqrt{\mu^{2}+\lambda^{2}}\right) e^{-i\langle\lambda / x\rangle}
$$


where $j_{(n-1) / 2}$ is the normalized Bessel function defined by

$$
\begin{aligned}
j_{(n-1) / 2}(x) & =2^{(n-1) / 2} \Gamma \frac{n+1}{2} \frac{J_{(n-1) / 2}(z)}{z^{(n-1) / 2}} \\
& =\Gamma \frac{n+1}{2} \sum_{k=0}^{+\infty} \frac{(-1)^{k}}{k ! \Gamma((2 k+1+n) / 2)}\left(\frac{z}{2}\right)^{2 k}
\end{aligned}
$$

with $J_{(n-1) / 2}$ the Bessel function of first kind and index $(n-1) / 2[9,15]$, and if $\lambda=$ $\left(\lambda_{1}, \ldots, \lambda_{n}\right) \in \mathbb{C}^{n}$ and $x=\left(x_{1}, \ldots, x_{n}\right) \in \mathbb{R}^{n}$, we put $\lambda^{2}=\lambda_{1}^{2}+\cdots+\lambda_{n}^{2}$ and $\langle\lambda / x\rangle=\lambda_{1} x_{1}+$ $\cdots+\lambda_{n} x_{n}$.

The normalized Bessel function $j_{(n-1) / 2}$ has the following Mehler integral representation:

$$
\forall r \in \mathbb{R}, \quad j_{(n-1) / 2}(r)=\frac{2 \Gamma((n+1) / 2)}{\sqrt{\pi} \Gamma(n / 2)} \int_{0}^{1}\left(1-t^{2}\right)^{n / 2-1} \cos (t r) d t,
$$

and therefore

$$
\forall k \in \mathbb{N}, \forall r \in \mathbb{R}, \quad\left|j_{(n-1) / 2}^{(k)}(r)\right| \leq 1
$$

Moreover, for all $\lambda \in \mathbb{C}$, the function

$$
r \longmapsto j_{(n-1) / 2}(\lambda r)
$$

is the unique solution of the differential equation

$$
\begin{aligned}
& l u(r)=-\lambda^{2} u(r), \\
& u(0)=1, \quad u^{\prime}(0)=0,
\end{aligned}
$$

where $l$ is the Bessel operator defined on $] 0,+\infty$ [ by (1.3).

On the other hand, the function $\varphi_{\mu, \lambda}$ is the unique solution of the system

$$
\begin{gathered}
D_{j} v(r, x)=-i \lambda_{j} v(r, x), \quad j=1,2, \ldots, n, \\
(l-\Delta) v(r, x)=-\mu^{2} v(r, x), \\
v(0,0)=1 ; \quad \frac{\partial v}{\partial r}(0, x)=0 \quad \forall x \in \mathbb{R}^{n},
\end{gathered}
$$

where $D_{j}=\partial / \partial x_{j}$, and $\Delta$ is the Laplacien operator on $\mathbb{R}^{n}$ :

$$
\Delta=\sum_{j=1}^{n} D_{j}^{2}
$$

Now let $\Gamma$ be the set

$$
\Gamma=\mathbb{R} \times \mathbb{R}^{n} \cup\left\{(i t, x) ;(t, x) \in \mathbb{R} \times \mathbb{R}^{n},|t| \leq\|x\|\right\} .
$$


We have for all $(\mu, \lambda) \in \Gamma$,

$$
\sup _{(r, x) \in \mathbb{R} \times \mathbb{R}^{n}}\left|\varphi_{\mu, \lambda}(r, x)\right|=1 .
$$

In the following, we will define a convolution product and the Fourier transform associated with the spherical mean operator. For this, we use the product formula for the functions $\varphi_{\mu, \lambda}$. For all $(r, x),(s, y) \in \mathbb{R} \times \mathbb{R}^{n}$,

$$
\varphi_{\mu, \lambda}(r, x) \varphi_{\mu, \lambda}(s, y)=\frac{\Gamma((n+1) / 2)}{\sqrt{\pi} \Gamma(n / 2)} \int_{0}^{\pi} \varphi_{\mu, \lambda}\left(\sqrt{r^{2}+s^{2}+2 r s \cos \theta}, x+y\right) \times(\sin \theta)^{n-1} \theta .
$$

We denote by (see [11])

(A) $d v(r, x)$ the measure defined on $\left[0,+\infty\left[\times \mathbb{R}^{n}\right.\right.$ by

$$
d \nu(r, x)=k_{n} r^{n} d r \otimes d x
$$

with

$$
k_{n}=\frac{1}{2^{(n-1) / 2} \Gamma((n+1) / 2)(2 \pi)^{n / 2}} ;
$$

(B) $L^{p}(d v), 1 \leq p \leq+\infty$, the space of measurable functions on $\left[0,+\infty\left[\times \mathbb{R}^{n}\right.\right.$, satisfying

$$
\begin{aligned}
& \|f\|_{p, v}=\left(\int_{\mathbb{R}^{n}} \int_{0}^{\infty}|f(r, x)|^{p} d \nu(r, x)\right)^{1 / p}<+\infty, \quad 1 \leq p<+\infty, \\
& \|f\|_{\infty, v}=\operatorname{esssup}_{(r, x) \in\left[0,+\infty\left[\times \mathbb{R}^{n}\right.\right.}|f(r, x)|<\infty, \quad p=+\infty ;
\end{aligned}
$$

(C) $d \gamma(\mu, \lambda)$ the measure defined on the set $\Gamma$ by

$$
\begin{aligned}
\int_{\Gamma} f(\mu, \lambda) d \gamma(\mu, \lambda)=k_{n}\{ & \int_{\mathbb{R}^{n}} \int_{0}^{\infty} f(\mu, \lambda)\left(\mu^{2}+\|\lambda\|^{2}\right)^{(n-1) / 2} \mu d \mu d \lambda \\
& \left.+\int_{\mathbb{R}^{n}} \int_{0}^{\|\lambda\|} f(i \mu, \lambda)\left(\|\lambda\|^{2}-\mu^{2}\right)^{(n-1) / 2} \mu d \mu d \lambda\right\} ;
\end{aligned}
$$

(D) $L^{p}(d \gamma), 1 \leq p \leq+\infty$, the space of measurable functions on $\Gamma$, satisfying

$$
\begin{aligned}
& \|f\|_{p, \gamma}=\left(\int_{\Gamma}|f(\mu, \lambda)|^{p} d \gamma(\mu, \lambda)\right)^{1 / p}<+\infty, \quad 1 \leq p<+\infty \\
& \|f\|_{\infty, \gamma}=\operatorname{esssup}_{(\mu, \lambda) \in \Gamma}|f(\mu, \lambda)|<\infty, \quad p=+\infty
\end{aligned}
$$

Definition 2.2. (i) The translation operator associated with the spherical mean operator is defined on $L^{1}(d \nu)$ by for all $(r, x),(s, y) \in\left[0,+\infty\left[\times \mathbb{R}^{n}\right.\right.$,

$$
\tau_{(r, x)} f(s, y)=\frac{\Gamma((n+1) / 2)}{\sqrt{\pi} \Gamma(n / 2)} \int_{0}^{\pi} f\left(\sqrt{r^{2}+s^{2}+2 r s \cos \theta}, x+y\right)(\sin \theta)^{n-1} d \theta .
$$


(ii) A convolution product associated with the spherical mean operator of $f, g \in$ $L^{1}(d v)$ is defined by for all $(r, x) \in\left[0,+\infty\left[\times \mathbb{R}^{n}\right.\right.$,

$$
f * g(r, x)=\int_{\mathbb{R}^{n}} \int_{0}^{\infty} f(s, y) \tau_{(r,-x)} \breve{g}(s, y) d \nu(s, y),
$$

where

$$
\breve{g}(r, x)=g(r,-x)
$$

We have the following properties.

(A) $\tau_{(r, x)} \varphi_{\mu, \lambda}(s, y)=\varphi_{\mu, \lambda}(r, x) \varphi_{\mu, \lambda}(s, y)$.

(B) If $f \in L^{p}(d v), 1 \leq p \leq+\infty$, then for all $(s, y) \in\left[0,+\infty\left[\times \mathbb{R}^{n}\right.\right.$, the function $\tau_{(s, y)} f \in$ $L^{p}(d \nu)$, and we have

$$
\left\|\tau_{(s, y)} f\right\|_{p, v} \leq\|f\|_{p, v} .
$$

(C) Let $1 \leq p, q, r \leq+\infty$ such that $1 / r=1 / p+1 / q-1$, then for all $f \in L^{p}(d \nu)$ and all $g \in L^{q}(d \nu)$, the function $f * g \in L^{r}(d \nu)$, and we have

$$
\|f * g\|_{r, v} \leq\|f\|_{p, v}\|g\|_{q, v} .
$$

Definition 2.3. The Fourier transform associated with the spherical mean operator is defined on $L^{1}(d \nu)$ by

$$
\forall(\mu, \lambda) \in \Gamma, \quad \mathscr{F} f(\mu, \lambda)=\int_{\mathbb{R}^{n}} \int_{0}^{\infty} f(r, x) \varphi_{\mu, \lambda}(r, x) d \nu(r, x) .
$$

We have the following properties.

(A) For all $(\mu, \lambda) \in \Gamma$,

$$
\mathscr{F} f(\mu, \lambda)=B o \tilde{\mathscr{F}} f(\mu, \lambda),
$$

where for all $(\mu, \lambda) \in \mathbb{R} \times \mathbb{R}^{n}$,

$$
\begin{gathered}
\tilde{\mathscr{F}} f(\mu, \lambda)=\int_{\mathbb{R}^{n}} \int_{0}^{\infty} f(r, x) j_{(n-1) / 2}(r \mu) e^{-i\langle\lambda / x\rangle} d \nu(r, x), \\
\forall(\mu, \lambda) \in \Gamma, \quad B f(\mu, \lambda)=f\left(\sqrt{\mu^{2}+\lambda^{2}}, \lambda\right) .
\end{gathered}
$$

(B) For $f \in L^{1}(d \nu)$ such that $\mathscr{F} f \in L^{1}(d \gamma)$, we have the inversion formula for $\mathscr{F}$ : for almost every $(r, x) \in\left[0,+\infty\left[\times \mathbb{R}^{n}\right.\right.$,

$$
f(r, x)=\iint_{\Gamma} \mathscr{F} f(\mu, \lambda) \overline{\varphi_{\mu, \lambda}(r, x)} d \gamma(\mu, \lambda) .
$$


(C) Let $f$ be in $L^{1}(d v)$. For all $(s, y) \in\left[0,+\infty\left[\times \mathbb{R}^{n}\right.\right.$, we have

$$
\forall(\mu, \lambda) \in \Gamma, \quad \mathscr{F}\left(\tau_{(s,-y)} f\right)(\mu, \lambda)=\varphi_{\mu, \lambda}(s, y) \mathscr{F} f(\mu, \lambda) .
$$

(D) For $f, g \in L^{1}(d v)$, we have

$$
\forall(\mu, \lambda) \in \Gamma, \quad \mathscr{F}(f * g)(\mu, \lambda)=\mathscr{F} f(\mu, \lambda) \mathscr{F} g(\mu, \lambda) .
$$

(E) For all $p \in[1,+\infty]$ and $f \in L^{p}(d v)$,

$$
B f \in L^{p}(d \gamma), \quad\|B f\|_{p, \gamma}=\|f\|_{p, v} .
$$

In particular, the mapping $B$ is an isometric isomorphism from $L^{2}(d v)$ onto $L^{2}(d \gamma)$. The mapping $\tilde{\mathscr{F}}$ is also an isometric isomorphism from $L^{2}(d v)$ onto itself. Consequently, the Fourier transform $\mathscr{F}$ is an isometric isomorphism from $L^{2}(d \nu)$ onto $L^{2}(d \gamma)$.

Thus,

$$
\forall f \in L^{2}(d v), \quad \mathscr{F} f \in L^{2}(d \gamma), \quad\|\mathscr{F} f\|_{2, \gamma}=\|f\|_{2, \nu} .
$$

Proposition 2.4 (see[11]). Let $f$ be in $L^{p}(d v)$, with $p \in[1,2]$. Then $\mathscr{F} f \in L^{p^{\prime}}(d \gamma)$, with $1 / p+1 / p^{\prime}=1$, and

$$
\left\|\mathscr{F}_{F} f\right\|_{p^{\prime}, \gamma} \leq\|f\|_{p, \nu} .
$$

We denote by

(A) $S_{*}\left(\mathbb{R} \times \mathbb{R}^{n}\right)$ the space of infinitely differentiable functions on $\mathbb{R} \times \mathbb{R}^{n}$, even with respect to the first variable, rapidly decreasing together with all their derivatives;

(B) $S_{*}(\Gamma)$ the space of infinitely differentiable functions on $\Gamma$, even with respect to the first variable, rapidly decreasing together with all their derivatives; that means for all $k_{1}, k_{2} \in \mathbb{N}$, for all $\alpha \in \mathbb{N}^{n}$,

$$
\sup \left\{\left(1+|\mu|^{2}+\|\lambda\|^{2}\right)^{k_{1}}\left|\left(\frac{\partial}{\partial \mu}\right)^{k_{2}} D_{\lambda}^{\alpha} f(\mu, \lambda)\right| ;(\mu, \lambda) \in \Gamma\right\}<+\infty,
$$

where

$$
\begin{aligned}
\frac{\partial f}{\partial \mu}(\mu, \lambda) & = \begin{cases}\frac{\partial}{\partial r}(f(r, \lambda)) & \text { if } \mu=r \in \mathbb{R}, \\
\frac{1}{i} \frac{\partial}{\partial t}(f(i t, \lambda)) & \text { if } \mu=i t,|t| \leq\|\lambda\|,\end{cases} \\
D_{\lambda}^{\alpha} & =\left(\frac{\partial}{\partial \lambda_{1}}\right)^{\alpha_{1}}\left(\frac{\partial}{\partial \lambda_{2}}\right)^{\alpha_{2}} \cdots\left(\frac{\partial}{\partial \lambda_{n}}\right)^{\alpha_{n}},
\end{aligned}
$$

(see [10]); 
(C) $S_{*}^{\prime}\left(\mathbb{R} \times \mathbb{R}^{n}\right)$ and $S_{*}^{\prime}(\Gamma)$ are, respectively, the dual spaces of $S_{*}\left(\mathbb{R} \times \mathbb{R}^{n}\right)$ and $S_{*}(\Gamma)$. Each of these spaces is equipped with its usual topology.

Remark 2.5. From [10], the Fourier transform $\mathscr{F}_{F}$ is a topological isomorphism from $S_{*}\left(\mathbb{R} \times \mathbb{R}^{n}\right)$ onto $S_{*}(\Gamma)$. The inverse mapping is given by for all $(r, x) \in \mathbb{R} \times \mathbb{R}^{n}$,

$$
\mathscr{F}^{-1} f(r, x)=\int_{\Gamma} f(\mu, \lambda) \overline{\varphi_{\mu, \lambda}(r, x)} d \gamma(\mu, \lambda) .
$$

Definition 2.6. The Fourier transform $\mathscr{F}$ is defined on $S_{*}^{\prime}\left(\mathbb{R} \times \mathbb{R}^{n}\right)$ by

$$
\forall T \in S_{*}^{\prime}\left(\mathbb{R} \times \mathbb{R}^{n}\right), \quad\langle\mathscr{F}(T), \varphi\rangle=\left\langle T, \mathscr{F}^{-1}(\varphi)\right\rangle, \quad \varphi \in S_{*}(\Gamma) .
$$

Since the Fourier transform $\mathscr{F}$ is an isomorphism from $S_{*}\left(\mathbb{R} \times \mathbb{R}^{n}\right)$ onto $S_{*}(\Gamma)$, we deduce that $\mathscr{F}$ is also an isomorphism from $S_{*}^{\prime}\left(\mathbb{R} \times \mathbb{R}^{n}\right)$ onto $S_{*}^{\prime}(\Gamma)$.

3. The space $M_{p}\left(\mathbb{R} \times \mathbb{R}^{n}\right)$

We denote by

(A) $L$ the partial differential operator defined by

$$
L=-\left(\frac{\partial^{2}}{\partial r^{2}}+\frac{n}{r} \frac{\partial}{\partial r}\right)-\sum_{j=0}^{n} \frac{\partial^{2}}{\partial x_{j}^{2}} ;
$$

(B) for $f \in L^{p}(d \nu), p \in[1, \infty], T_{f}$ is the element of $S_{*}^{\prime}\left(\mathbb{R} \times \mathbb{R}^{n}\right)$ defined by

$$
\left\langle T_{f}, \varphi\right\rangle=\int_{\mathbb{R}^{n}} \int_{0}^{\infty} f(r, x) \varphi(r, x) d \nu(r, x), \quad \varphi \in S_{*}\left(\mathbb{R} \times \mathbb{R}^{n}\right) ;
$$

(C) for $g \in L^{p}(d \gamma), p \in[1, \infty], T_{g}$ is the element of $S_{*}^{\prime}(\Gamma)$ defined by

$$
\left\langle T_{g}, \psi\right\rangle=\int_{\Gamma} g(\mu, \lambda) \psi(\mu, \lambda) d \gamma(\mu, \lambda), \quad \psi \in S_{*}(\Gamma) .
$$

From Proposition 2.4 and Remark 2.5, we deduce that for all $f \in L^{p}(d v), 1 \leq p \leq 2$, $\mathscr{F} f$ belongs to the space $L^{p^{\prime}}(d \gamma)$ and we have

$$
\mathscr{F}\left(T_{f}\right)=T_{\mathscr{F}(\breve{f})} \cdot
$$

Definition 3.1. Let $p \in[1, \infty]$. We define $M_{p}\left(\mathbb{R} \times \mathbb{R}^{n}\right)$ to be the set of measurable functions $f$ on $\mathbb{R} \times \mathbb{R}^{n}$, even with respect to the first variable, and such that for all $k \in \mathbb{N}$ there exists $g_{k} \in L^{p}(d v)$ satisfying

$$
L^{k} T_{f}=T_{g_{k}}
$$

The space $\mathcal{M}_{p}\left(\mathbb{R} \times \mathbb{R}^{n}\right)$ is equipped with the topology generated by the family of norms

$$
\gamma_{m, p}(f)=\max _{0 \leq k \leq m}\left\|g_{k}\right\|_{p, v}, \quad m \in \mathbb{N},
$$


where $g_{k}, k \in \mathbb{N}$, is the function given by the relation (3.5). Let

$$
\begin{aligned}
& d_{p}: M_{p}\left(\mathbb{R} \times \mathbb{R}^{n}\right) \times M_{p}\left(\mathbb{R} \times \mathbb{R}^{n}\right) \longrightarrow[0, \infty[ \\
& (f, g) \longmapsto d_{p}(f, g)=\sum_{m=0}^{\infty} \frac{1}{2^{m}} \frac{\gamma_{m, p}(f-g)}{1+\gamma_{m, p}(f-g)} .
\end{aligned}
$$

Then $d_{p}$ is a distance on $\mu_{p}\left(\mathbb{R} \times \mathbb{R}^{n}\right)$. Moreover the sequence $\left(f_{k}\right)_{k \in \mathbb{N}}$ converges to 0 in $\left(\mathcal{M}_{p}\left(\mathbb{R} \times \mathbb{R}^{n}\right), d_{p}\right)$ if and only if

$$
\forall m \in \mathbb{N}, \quad \gamma_{m, p}\left(f_{k}\right) \underset{k \rightarrow \infty}{\longrightarrow} 0
$$

In the following, we will give some properties of the space $\mu_{p}\left(\mathbb{R} \times \mathbb{R}^{n}\right)$.

Proposition 3.2. $\left(M_{p}\left(\mathbb{R} \times \mathbb{R}^{n}\right), d_{p}\right)$ is a Frechet space.

Proof. Let $\left(f_{m}\right)_{m \in \mathbb{N}}$ be a Cauchy sequence in $\left(\mu_{p}\left(\mathbb{R} \times \mathbb{R}^{n}\right), d_{p}\right)$ and let $\left(g_{m, k}\right)_{m \in \mathbb{N}} \subset L^{p}(d \nu)$ such that

$$
L^{k} T_{f_{m}}=T_{g_{m, k}}, \quad k \in \mathbb{N}
$$

Then for all $k \in \mathbb{N},\left(g_{m, k}\right)_{m \in \mathbb{N}}$ is a Cauchy sequence in $L^{p}(d \nu)$. We put

$$
\begin{gathered}
f=g_{0}=\lim _{m \rightarrow \infty} f_{m}, \\
g_{k}=\lim _{m \rightarrow \infty} g_{m, k}, \quad k \in \mathbb{N}^{*},
\end{gathered}
$$

in $L^{p}(d \nu)$. Thus

$$
\forall k \in \mathbb{N}, \quad T_{g_{m, k}} \underset{m \rightarrow \infty}{\longrightarrow} T_{g_{k}}
$$

in $S_{*}^{\prime}\left(\mathbb{R} \times \mathbb{R}^{n}\right)$. Since $L^{k}$ is a continuous operator from $S_{*}^{\prime}\left(\mathbb{R} \times \mathbb{R}^{n}\right)$ into itself, we deduce that

$$
L^{k} T_{f_{m}} \underset{m \rightarrow \infty}{\longrightarrow} L^{k} T_{f}
$$

in $S_{*}^{\prime}\left(\mathbb{R} \times \mathbb{R}^{n}\right)$.

From relations (3.9) and (3.11), we deduce that

$$
\forall k \in \mathbb{N}, \quad L^{k} T_{f}=T_{g_{k}} .
$$

This proves that $f \in M_{p}\left(\mathbb{R} \times \mathbb{R}^{n}\right)$ and

$$
f_{m} \underset{m \rightarrow \infty}{\longrightarrow} f
$$

in $\left(M_{p}\left(\mathbb{R} \times \mathbb{R}^{n}\right), d_{p}\right)$. 
Proposition 3.3. Let $p \in[1,2]$ and $f \in \mathcal{M}_{p}\left(\mathbb{R} \times \mathbb{R}^{n}\right)$, then

(i) for all $k \in \mathbb{N}$, the function

$$
(\mu, \lambda) \longrightarrow\left(1+\mu^{2}+2\|\lambda\|^{2}\right)^{k} \mathscr{F}_{F}(f)(\mu, \lambda)
$$

belongs to the space $L^{p^{\prime}}(d \gamma)$ with $p^{\prime}=p /(p-1)$;

(ii) $\mathcal{M}_{p}\left(\mathbb{R} \times \mathbb{R}^{n}\right) \cap \mathscr{C}_{*}\left(\mathbb{R} \times \mathbb{R}^{n}\right) \subset \mathscr{E}_{*}\left(\mathbb{R} \times \mathbb{R}^{n}\right)$, where $\mathscr{C}_{*}\left(\mathbb{R} \times \mathbb{R}^{n}\right)$ is the space of continuous functions on $\mathbb{R} \times \mathbb{R}^{n}$ even with respect to the first variable.

Proof. (i) Let $f \in M_{p}\left(\mathbb{R} \times \mathbb{R}^{n}\right), 1 \leq p \leq 2$, and $g_{k} \in L^{p}(d v)$ such that

$$
L^{k} T_{f}=T_{g_{k}} \quad k \in \mathbb{N} .
$$

From relation (3.4), we have

$$
\mathscr{F}\left(T_{g_{k}}\right)=T_{\mathscr{F}\left(\breve{g}_{k}\right)},
$$

which gives

$$
\mathscr{F}\left(L^{k} T_{f}\right)=T_{\mathscr{F}\left(\breve{g}_{k}\right)} .
$$

On the other hand

$$
\mathscr{F}\left(L^{k} T_{f}\right)=\left(\mu^{2}+2\|\lambda\|^{2}\right)^{k} \mathscr{F}\left(T_{f}\right)=T_{\left(\mu^{2}+2\|\lambda\|^{2}\right)^{k \mathscr{F}}(\breve{f})},
$$

hence

$$
\left(\mu^{2}+2\|\lambda\|^{2}\right)^{k} \mathscr{F}_{(}(f)=\mathscr{F}\left(g_{k}\right) .
$$

This equality, together with the fact that the function $\mathscr{F}\left(g_{k}\right)$ belongs to the space $L^{p^{\prime}}(d \nu)$ implies (i).

(ii) Let $f \in \mathcal{M}_{p}\left(\mathbb{R} \times \mathbb{R}^{n}\right) \cap \mathscr{C}_{*}\left(\mathbb{R} \times \mathbb{R}^{n}\right)$. From the assertion (i) and relations (2.26) and (2.31), we deduce that for all $k \in \mathbb{N}$, the function

$$
(r, x) \longrightarrow\left(r^{2}+\|x\|^{2}\right)^{k} \tilde{\mathscr{F}}(f)
$$

belongs to the space $L^{p^{\prime}}(d \nu)$, in particular $\tilde{\mathscr{F}}(f) \in L^{1}(d \nu) \cap L^{2}(d \nu)$.

On the other hand, the transform $\tilde{\mathscr{F}}$ is an isometric isomorphism from $L^{2}(d v)$ onto itself, then from the inversion formula for $\tilde{\mathscr{F}}$ and using the continuity of the function $f$, we have for all $(r, x) \in \mathbb{R} \times \mathbb{R}^{n}$,

$$
f(r, x)=\int_{\mathbb{R}^{n}} \int_{0}^{\infty} \tilde{\mathscr{F}}_{\mathcal{F}} f(\mu, \lambda) j_{(n-1) / 2}(r \mu) e^{i\langle\lambda / x\rangle} d \nu(\mu, \lambda) .
$$

Consequently, (ii) follows from relation (2.7) and the fact that for all $k \in \mathbb{N}, \alpha \in \mathbb{N}^{n}$, the function

$$
(\mu, \lambda) \longrightarrow \mu^{k} \lambda^{\alpha} \widetilde{\mathscr{F}}(\mu, \lambda)
$$

belongs to the space $L^{1}(d \nu)$. 
Proposition 3.4. Let $p \in[1,2]$, then, for all $r \in[2, \infty]$,

$$
\mu_{p}\left(\mathbb{R} \times \mathbb{R}^{n}\right) \cap \mathscr{C}_{*}\left(\mathbb{R} \times \mathbb{R}^{n}\right) \subset \mathcal{M}_{r}\left(\mathbb{R} \times \mathbb{R}^{n}\right)
$$

Proof. Let $f \in \mathcal{M}_{p}\left(\mathbb{R} \times \mathbb{R}^{n}\right) \cap \mathscr{C}_{*}\left(\mathbb{R} \times \mathbb{R}^{n}\right), p \in[1,2], r \geq 2$, and $r^{\prime}=r /(r-1)$. From Proposition 3.3, we deduce that $f \in \mathscr{E}_{*}\left(\mathbb{R} \times \mathbb{R}^{n}\right)$ and for all $k \in \mathbb{N}$, the function (3.21) belongs to the space $L^{p^{\prime}}(d \nu)$. By applying Holder's inequality, it follows that this last function belongs to the space $L^{r^{\prime}}(d v)$. On the other hand, for all $(r, x) \in \mathbb{R} \times \mathbb{R}^{n}$,

$$
\begin{aligned}
L^{k} f(r, x) & =\int_{\mathbb{R}^{n}} \int_{0}^{\infty}\left(\mu^{2}+\|\lambda\|^{2}\right)^{k} \tilde{\mathscr{F}}(f)(\mu, \lambda) j_{(n-1) / 2}(r \mu) e^{i\langle\lambda / x\rangle} d \nu(\mu, \lambda) \\
& =\widetilde{\mathscr{F}}\left(\left(\mu^{2}+\|\lambda\|^{2}\right)^{k} \tilde{\mathscr{F}}(\breve{f})\right)(r, x) .
\end{aligned}
$$

From Proposition 2.4 and the fact that

$$
\|\mathscr{F}(g)\|_{r, \gamma}=\|\tilde{\mathscr{F}}(g)\|_{r, v}, \quad g \in L^{r^{\prime}}(d v)
$$

we deduce that, for all $k \in \mathbb{N}$, the function $L^{k} f$ belongs to the space $L^{r}(d v)$.

\section{The dual space $M_{p}^{\prime}\left(\mathbb{R} \times \mathbb{R}^{n}\right)$}

In this section, we will give a new characterization of the dual space $M_{p}^{\prime}\left(\mathbb{R} \times \mathbb{R}^{n}\right)$ of $\mathcal{M}_{p}\left(\mathbb{R} \times \mathbb{R}^{n}\right)$. We recall that for every $f \in \mathcal{M}_{p}\left(\mathbb{R} \times \mathbb{R}^{n}\right)$, the family $\left\{V_{m, p, \varepsilon}(f), m \in \mathbb{N}, \varepsilon>0\right\}$ is a basic of neighborhoods of $f$ in $\left(M_{p}\left(\mathbb{R} \times \mathbb{R}^{n}\right), d_{p}\right)$, where

$$
V_{m, p, \varepsilon}(f)=\left\{g \in M_{p}\left(\mathbb{R} \times \mathbb{R}^{n}\right), \gamma_{m, p}(f-g)<\varepsilon\right\} .
$$

In addition, $T \in M_{p}^{\prime}\left(\mathbb{R} \times \mathbb{R}^{n}\right)$ if and only if there exist $m \in \mathbb{N}$ and $c>0$ such that

$$
\forall f \in M_{p}\left(\mathbb{R} \times \mathbb{R}^{n}\right), \quad|\langle T, f\rangle| \leq c \gamma_{m, p}(f) .
$$

For $f \in L^{p^{\prime}}(d \nu)$ and $\varphi \in M_{p}\left(\mathbb{R} \times \mathbb{R}^{n}\right)$, we put

$$
\left\langle L^{k}\left(T_{f}\right), \varphi\right\rangle=\int_{\mathbb{R}^{n}} \int_{0}^{\infty} f(r, x) \psi_{k}(r, x) d \nu(r, x)
$$

with $L^{k} T_{\varphi}=T_{\psi_{k}}$. Then

$$
\left|\left\langle L^{k}\left(T_{f}\right), \varphi\right\rangle\right| \leq\|f\|_{p^{\prime}, v}\left\|\psi_{k}\right\|_{p, v} \leq\|f\|_{p^{\prime}, v} \gamma_{k, p}(\varphi) .
$$

This proves that for all $f \in L^{p^{\prime}}(d v)$ and $k \in \mathbb{N}$, the functional $L^{k} T_{f}$ defined by the relation (4.3) belongs to the space $M_{p}^{\prime}\left(\mathbb{R} \times \mathbb{R}^{n}\right)$.

In the following, we will prove that every element of $M_{p}^{\prime}\left(\mathbb{R} \times \mathbb{R}^{n}\right)$ is also of this type. 
Theorem 4.1. Let $T \in S_{*}^{\prime}\left(\mathbb{R} \times \mathbb{R}^{n}\right)$. Then $T \in \mathcal{M}_{p}^{\prime}\left(\mathbb{R} \times \mathbb{R}^{n}\right), 1 \leq p<\infty$, if and only if there exist $m \in \mathbb{N}$ and $\left\{f_{0}, \ldots, f_{m}\right\} \subset L^{p^{\prime}}(d v)$ such that

$$
T=\sum_{k=0}^{m} L^{k} T_{f_{k}}
$$

where $L^{k} T_{f_{k}}$ is given by relation (4.3).

Proof. It is clear that if

$$
T=\sum_{k=0}^{m} L^{k} T_{f_{k}}, \quad\left\{f_{0}, \ldots, f_{m}\right\} \subset L^{p^{\prime}}(d \nu),
$$

then $T$ belongs to the space $M_{p}^{\prime}\left(\mathbb{R} \times \mathbb{R}^{n}\right)$.

Conversely, suppose that $T \in \mathcal{M}_{p}^{\prime}\left(\mathbb{R} \times \mathbb{R}^{n}\right)$. From relation (4.2) there exist $m \in \mathbb{N}$ and $c>0$ such that

$$
\forall \varphi \in M_{p}\left(\mathbb{R} \times \mathbb{R}^{n}\right), \quad|\langle T, \varphi\rangle| \leq c \gamma_{m, p}(\varphi)
$$

Let

$$
\left(L^{p}(d \nu)\right)^{m+1}=\left\{\left(f_{0}, \ldots, f_{m}\right), f_{k} \in L^{p}(d \nu), 0 \leq k \leq m\right\}
$$

equipped with the norm

$$
\left\|\left(f_{0}, \ldots, f_{m}\right)\right\|_{\left(L^{p}(d v)\right)^{m+1}}=\max _{0 \leq k \leq m}\left\|f_{k}\right\|_{p, v^{*}}
$$

We consider the mappings

$$
\begin{gathered}
\mathscr{A}: \mathcal{M}_{p}\left(\mathbb{R} \times \mathbb{R}^{n}\right) \longrightarrow\left(L^{p}(d \nu)\right)^{m+1}, \\
\varphi \longmapsto\left(\varphi, g_{1}, \ldots, g_{m}\right),
\end{gathered}
$$

where

$$
\begin{gathered}
L^{k} T_{\varphi}=T_{g_{k}}, \quad k \geq 1, \\
\mathscr{B}: \operatorname{Im}(\mathscr{A}) \longrightarrow \mathbb{C}, \\
\mathscr{B}(\mathscr{A} \varphi)=\langle T, \varphi\rangle .
\end{gathered}
$$

From relation (4.2) we deduce that

$$
|\mathscr{B} \mathscr{A}(\varphi)|=|\langle T, \varphi\rangle| \leq\left. c|| \mathscr{A}(\varphi)\right|_{\left(L^{p}(d v)\right)^{m+1}} .
$$

This means that $\mathscr{B}$ is a continuous functional on the subspace $\operatorname{Im}(\mathscr{A})$ of the space $\left(L^{p}(d \nu)\right)^{m+1}$. From Hahn-Banach theorems, there exists a continuous extension of $\mathscr{B}$ to $\left(L^{p}(d v)\right)^{m+1}$, denoted again by $\mathscr{B}$. 
By Riez's theorem there exist $\left(f_{0}, \ldots, f_{m}\right) \in\left(L^{p^{\prime}}(d \nu)\right)^{m+1}$ such that for all $\left(\varphi_{0}, \ldots, \varphi_{m}\right) \in$ $\left(L^{p}(d \nu)\right)^{m+1}$,

$$
\mathscr{B}\left(\varphi_{0}, \ldots, \varphi_{m}\right)=\sum_{k=0}^{m} \int_{\mathbb{R}^{n}} \int_{0}^{\infty} f_{k}(r, x) \varphi_{k}(r, x) d \nu(r, x) .
$$

By means of relation (4.3), we deduce that for $\varphi \in \mathcal{M}_{p}\left(\mathbb{R} \times \mathbb{R}^{n}\right)$, we have

$$
\langle T, \varphi\rangle=\sum_{k=0}^{m} \int_{\mathbb{R}^{n}} \int_{0}^{\infty} f_{k}(r, x) \varphi_{k}(r, x) d \nu(r, x)=\sum_{k=0}^{m}\left\langle L^{k} T_{f_{k}}, \varphi\right\rangle .
$$

This completes the proof of Theorem 4.1.

Proposition 4.2. Let $p \geq 2$. Then for all $T \in M_{p}^{\prime}\left(\mathbb{R} \times \mathbb{R}^{n}\right)$, there exist $m \in \mathbb{N}$ and $F \in$ $L^{p}(d \gamma)$ such that

$$
\mathscr{F}(T)=T_{\left(1+\mu^{2}+2\|\lambda\|^{2}\right)^{m} F} .
$$

Proof. Let $T \in M_{p}^{\prime}\left(\mathbb{R} \times \mathbb{R}^{n}\right)$. From Theorem 4.1 there exist $m \in \mathbb{N}$ and $\left(f_{0}, \ldots, f_{m}\right) \in$ $\left(L^{p^{\prime}}(d \nu)\right)^{m+1}, p^{\prime}=p /(p-1)$, such that

$$
T=\sum_{k=0}^{m} L^{k} T_{f_{k}}
$$

Consequently

$$
\mathscr{F}(T)=\sum_{k=0}^{m} \mathscr{F}_{\mathcal{F}}\left(L^{k} T_{f_{k}}\right)=\sum_{k=0}^{m}\left(\mu^{2}+2\|\lambda\|^{2}\right)^{k} \mathscr{F}_{(}\left(T_{f_{k}}\right) .
$$

By using relation (3.4) we get (4.15), where

$$
F=\sum_{k=0}^{m} \frac{\left(\mu^{2}+2\|\lambda\|^{2}\right)^{k}}{\left(1+\mu^{2}+2\|\lambda\|^{2}\right)^{m}} \mathscr{F}\left(\check{f}_{k}\right)
$$

which proves the result.

Proposition 4.3. Let $T \in S_{*}^{\prime}\left(\mathbb{R} \times \mathbb{R}^{n}\right)$, then $T \in M_{2}^{\prime}\left(\mathbb{R} \times \mathbb{R}^{n}\right)$ if and only if there exist $m \in \mathbb{N}$ and $F \in L^{2}(d \gamma)$ such that (4.15) holds.

Proof. From Proposition 4.2, we deduce that if $T \in M_{2}^{\prime}\left(\mathbb{R} \times \mathbb{R}^{n}\right)$, then there exist $m \in \mathbb{N}$ and $F \in L^{2}(d \gamma)$ verifying (4.15). Conversely, suppose that (4.15) holds with $F \in L^{2}(d \gamma)$. Since $\mathscr{F}$ is an isometric isomorphism from $L^{2}(d \nu)$ onto $L^{2}(d \gamma)$, then there exists $G \in$ $L^{2}(d \nu)$ such that $\mathscr{F}(G)=F$ and from relation (3.4) we have

$$
\mathscr{F}\left(T_{\breve{G}}\right)=T_{F} .
$$


Consequently

$$
\mathscr{F}(T)=\mathscr{F}\left((I+L)^{m} T_{\breve{G}}\right),
$$

thus

$$
T=\sum_{k=0}^{m} C_{m}^{k} L^{k} T_{\breve{G}}
$$

and Theorem 4.1 implies that $T \in \mathcal{M}_{2}^{\prime}\left(\mathbb{R} \times \mathbb{R}^{n}\right)$.

We denote by

(A) $\mathscr{D}_{*}\left(\mathbb{R} \times \mathbb{R}^{n}\right)$ the space of infinitely differentiable functions on $\mathbb{R} \times \mathbb{R}^{n}$, even with respect to the first variable and with compact support, equipped with its usual topology;

(B) for $a>0, \mathscr{D}_{*, a}\left(\mathbb{R} \times \mathbb{R}^{n}\right)$ the subspace of $\mathscr{D}_{*}\left(\mathbb{R} \times \mathbb{R}^{n}\right)$ consisting of function $f$ such that supp $f \subset B(0, a)=\left\{(r, x) \in \mathbb{R} \times \mathbb{R}^{n}, r^{2}+\|x\|^{2} \leq a^{2}\right\}$

(C) for $a>0, \mathscr{D}_{*, a}^{\prime}\left(\mathbb{R} \times \mathbb{R}^{n}\right)$ the dual space of $\mathscr{D}_{*, a}\left(\mathbb{R} \times \mathbb{R}^{n}\right)$;

(D) for $a>0$ and $m \in \mathbb{N}, \mathcal{W}_{a}^{m}\left(\mathbb{R} \times \mathbb{R}^{n}\right)$ the space of function $f: \mathbb{R} \times \mathbb{R}^{n} \rightarrow \mathbb{C}$ of class $C^{2 m}$ on $\mathbb{R} \times \mathbb{R}^{n}$, even with respect to the first variable and with support in $B(0, a)$, normed by

$$
N_{\infty, m}(f)=\max _{0 \leq k \leq m}\left\|L^{k}(f)\right\|_{\infty, v}
$$

Proposition 4.4. Let $a>0$ and $m \in \mathbb{N}$. Then there exists $p_{o} \in \mathbb{N}$ such that for every $p \in \mathbb{N}$, $p \geq p_{o}$, it is possible to find $\varphi_{p} \in W_{a}^{m}\left(\mathbb{R} \times \mathbb{R}^{n}\right)$ and $\psi_{p} \in \mathscr{D}_{*, a}\left(\mathbb{R} \times \mathbb{R}^{n}\right)$ satisfying

$$
\delta=(I+L)^{p} T_{\varphi_{p}}+T_{\psi_{p}}
$$

in $S_{*}^{\prime}\left(\mathbb{R} \times \mathbb{R}^{n}\right)$.

Proof. Let $p \geq n+1$ and $g_{p}$ the function defined by

$$
\forall(\mu, \lambda) \in \mathbb{R} \times \mathbb{R}^{n}, \quad g_{p}(\mu, \lambda)=\tilde{\mathscr{F}}\left(\frac{1}{\left(1+r^{2}+\|x\|^{2}\right)^{p}}\right)(\mu, \lambda) .
$$

Using relation (2.7), we deduce that there exists $p_{o} \in \mathbb{N}$ such that for all $p \geq p_{o}$ the function $g_{p}$ is of class $C^{2 m}$ on $\mathbb{R} \times \mathbb{R}^{n}$ (e.g., we can choose $p_{o}=3 n+1+2 m$ ).

Now, we prove that the function $g_{p}$ is infinitely differentiable on $\mathbb{R} \times \mathbb{R}^{n} \backslash\{(0, \ldots, 0)\}$. The function $g_{p}$ can be written as

$$
g_{p}(\mu, \lambda)=\frac{1}{2^{n-1 / 2} \Gamma(n+1 / 2)} \int_{0}^{\infty} \frac{1}{\left(1+s^{2}\right)^{p}} j_{n-1 / 2}\left(s \sqrt{\mu^{2}+\|\lambda\|^{2}}\right) s^{2 n} d s
$$


By relation (2.6) and Fubini's theorem we get

$$
\begin{aligned}
g_{p}(\mu, \lambda) & =\frac{1}{2^{n-1 / 2} \sqrt{\pi} \Gamma(n)} \int_{-1}^{1}\left(1-t^{2}\right)^{n-1}\left[\int_{0}^{\infty} \frac{\cos \left(t s \sqrt{\mu^{2}+\|\lambda\|^{2}}\right)}{\left(1+s^{2}\right)^{p}} s^{2 n} d s\right] d t \\
& =\frac{1}{2^{n-3 / 2} \sqrt{\pi} \Gamma(n)} \int_{0}^{1}\left(1-t^{2}\right)^{n-1} h_{p}\left(t \sqrt{\mu^{2}+\|\lambda\|^{2}}\right) d t
\end{aligned}
$$

where

$$
h_{p}(u)=\int_{0}^{\infty} \frac{\cos (s u)}{\left(1+s^{2}\right)^{p}} s^{2 n} d s=\frac{1}{2} \int_{-\infty}^{\infty} \frac{e^{i s u}}{\left(1+s^{2}\right)^{p}} s^{2 n} d s .
$$

By standard calculus, we have

$$
\int_{0}^{\infty} \frac{\cos (s u)}{\left(1+s^{2}\right)^{p}} s^{2 n} d s=e^{-u} P(u)
$$

with

$$
P(u)=\frac{\pi}{2^{2 p-1}} \sum_{k=0}^{p-1} \frac{C_{2 p-2-k}^{p-1}}{k !}(2 u)^{k} .
$$

On the other hand, we have

$$
h_{p}(u)=(-1)^{n}\left(\frac{d}{d u}\right)^{2 n}\left(\frac{1}{2} \int_{-\infty}^{\infty} \frac{e^{i s u}}{\left(1+s^{2}\right)^{p}} d s\right),
$$

then, we get

$$
\forall u \geq 0, \quad h_{p}(u)=Q_{p}(u) e^{-u},
$$

where $Q_{p}$ is a real polynomial. Since $h_{p}$ is an even function on $\mathbb{R}$, then we deduce that

$$
\forall u \in \mathbb{R}, \quad h_{p}(u)=k_{p}(|u|),
$$

where $k_{p}$ is the infinitely differentiable function defined on $\mathbb{R}$ by

$$
k_{p}(u)=Q_{p}(u) e^{-u}
$$

Now, the function

$$
u \longrightarrow F_{p}(u)=\frac{1}{2^{n-3 / 2} \sqrt{\pi} \Gamma(n)} \int_{0}^{1}\left(1-t^{2}\right)^{n-1} k_{p}(t u) d t
$$

is infinitely differentiable on $\mathbb{R}$ and we have

$$
g_{p}(\mu, \lambda)=F_{p}\left(\sqrt{\mu^{2}+\|\lambda\|^{2}}\right)
$$


This shows that the function $g_{p}$ is infinitely differentiable on $\mathbb{R} \times \mathbb{R}^{n} \backslash\{(0, \ldots, 0)\}$, even with respect to the first variable.

Let $\gamma \in \mathscr{D}_{*, a}\left(\mathbb{R} \times \mathbb{R}^{n}\right)$ such that

$$
\forall(r, x) \in \mathbb{R} \times \mathbb{R}^{n}, \quad r^{2}+x^{2} \leq \frac{a^{2}}{4}, \quad \gamma(r, x)=1 .
$$

Since $(I+L)^{p} T_{g_{p}}=\delta$, we get

$$
\gamma(I+L)^{p} T_{g_{p}}=(I+L)^{p} T_{g_{p}}=\delta .
$$

On the other hand, by using the fact that the function $g_{p}$ is infinitely differentiable on $\mathbb{R} \times \mathbb{R}^{n} \backslash\{(0, \ldots, 0)\}$, we deduce that the function

$$
\varphi_{p}(r, x)=(\gamma-1)(I+L)^{p} g_{p}+(I+L)^{p}\left((1-\gamma) g_{p}\right)
$$

belongs to the space $\mathscr{D}_{*, a}\left(\mathbb{R} \times \mathbb{R}^{n}\right)$.

Moreover, from relation (4.37), we have

$$
T_{(\gamma-1)(I+L)^{p} g_{p}}=(\gamma-1)(I+L)^{p} T_{g_{p}}=0,
$$

and this implies by using relation (4.38) that

$$
T_{\varphi_{p}}=T_{(I+L)^{p}\left((1-\gamma) g_{p}\right)}=(I+L)^{p} T_{\left((1-\gamma) g_{p}\right)} .
$$

Hence,

$$
T_{\varphi_{p}}+(I+L)^{p} T_{\gamma g_{p}}=(I+L)^{p} T_{g_{p}}=\delta,
$$

and this completes the proof of the proposition by taking $\psi_{p}=\gamma g_{p}$.

To prove the main result of this section, that is, Theorem 4.7, we will define some new families of norms on the space $\mathscr{D}_{*, a}\left(\mathbb{R} \times \mathbb{R}^{n}\right)$. We use these norms to prove that the elements of all bounded subset $B^{\prime} \subset \mathscr{D}_{*, a}^{\prime}\left(\mathbb{R} \times \mathbb{R}^{n}\right)$ can be continuously extended on the space $\mathcal{W}_{a}^{m}\left(\mathbb{R} \times \mathbb{R}^{n}\right)$.

For $f \in \mathscr{D}_{*, a}\left(\mathbb{R} \times \mathbb{R}^{n}\right), a>0$,

(A) $P_{m}(f)=\max _{k+|\alpha| \leq m}\left\|(\partial / \partial r)^{k} D^{\alpha} f\right\|_{\infty, \nu}$,

(B) $\widetilde{P}_{m}(f)=\max _{k+|\alpha| \leq m}\left\|l^{k} D^{\alpha} f\right\|_{\infty, v}$,

(C) $N_{p, m}(f)=\max _{0 \leq k \leq m}\left\|L^{k}(f)\right\|_{p, v}, p \in[1, \infty]$,

where $l$ is defined by relation (1.3).

Lemma 4.5. (i) For all $m \in \mathbb{N}$, there exists $c_{1}>0$ such that

$$
\forall \varphi \in \mathscr{D}_{*, a}\left(\mathbb{R} \times \mathbb{R}^{n}\right), \quad P_{m}(\varphi) \leq c_{1} \widetilde{P}_{m}(\varphi) .
$$

(ii) For all $m \in \mathbb{N}$, there exist $c_{2}>0$ and $m^{\prime} \in \mathbb{N}$ such that

$$
\forall \varphi \in \mathscr{D}_{*, a}\left(\mathbb{R} \times \mathbb{R}^{n}\right), \quad \widetilde{P}_{m}(\varphi) \leq c_{2} N_{p, m^{\prime}}(\varphi) .
$$


Proof. (i) Let $m \in \mathbb{N}$, and $\varphi \in \mathscr{D}_{*, a}\left(\mathbb{R} \times \mathbb{R}^{n}\right)$. By induction on $k$ we have

$$
\left(\frac{\partial}{\partial r}\right)^{k} D^{\alpha} \varphi(r, x)=\sum_{s=0}^{k} P_{s}(r)\left(\frac{\partial}{\partial r^{2}}\right)^{s} D^{\alpha} \varphi(r, x),
$$

where $P_{s}$ is a real polynomial. On the other hand, and also by induction, we deduce that for all $s \geq 1$,

$$
\left(\frac{\partial}{\partial r^{2}}\right)^{s} D^{\alpha} \varphi(r, x)=\int_{0}^{1} \cdots \int_{0}^{1} l^{s} D^{\alpha} \varphi\left(r t_{1}, \ldots, t_{s}, x\right) t_{1}^{n+2(s-1)}, \ldots, t_{s}^{n} d t_{1}, \ldots, d t_{s} .
$$

From relations (4.44) and (4.45), it follows that there exists $c_{a, m}>0$ satisfying

$$
P_{m}(\varphi) \leq c_{a, m} \widetilde{P}_{m}(\varphi)
$$

(ii) Let $p \in[1, \infty], m \in \mathbb{N}$, and $m_{1} \in \mathbb{N}$ such that

$$
\left\|\frac{1}{\left(1+\mu^{2}+\|\lambda\|^{2}\right)^{m_{1}}}\right\|_{1, v}<\infty
$$

then, for all $(k, \alpha) \in \mathbb{N} \times \mathbb{N}^{n}, k+|\alpha| \leq m$, we have

$$
\begin{aligned}
& \left\|l^{k} D^{\alpha} \varphi\right\|_{\infty, v}=\left\|\tilde{\mathscr{F}}^{-1}\left(\tilde{\mathscr{F}}\left(l^{k} D^{\alpha} \varphi\right)\right)\right\|_{\infty, v} \\
& \leq\left\|\tilde{\mathscr{F}}\left(l^{k} D^{\alpha} \varphi\right)\right\|_{1, v} \\
& \leq\left\|\mu^{2 k} \lambda^{\alpha} \tilde{\mathscr{F}}(\varphi)\right\|_{1, v} \\
& \leq\left\|\left(1+\mu^{2}+\|\lambda\|^{2}\right)^{m} \tilde{\mathscr{F}}(\varphi)\right\|_{1, v} \\
& =\left\|\frac{1}{\left(1+\mu^{2}+\|\lambda\|^{2}\right)^{m_{1}}} \tilde{\mathscr{F}}\left((I+L)^{m+m_{1}} \varphi\right)\right\|_{1, v} \\
& \leq\left\|\frac{1}{\left(1+\mu^{2}+\|\lambda\|^{2}\right)^{m_{1}}}\right\|\left\|_{1, \nu}\right\| \tilde{\mathscr{F}}\left((I+L)^{m+m_{1}} \varphi\right) \|_{\infty, \nu} \\
& \leq\left\|\frac{1}{\left(1+\mu^{2}+\|\lambda\|^{2}\right)^{m_{1}}}\right\|\left\|_{1, \nu}\right\|(I+L)^{m+m_{1}} \varphi \|_{1, \nu} \text {, }
\end{aligned}
$$

and by Holder's inequality, we get

$$
\begin{aligned}
\left\|l^{k} D^{\alpha} \varphi\right\|_{\infty, \nu} & \leq\left\|\frac{1}{\left(1+\mu^{2}+\|\lambda\|^{2}\right)^{m_{1}}}\right\|_{1, \nu}(\nu(B(0, a)))^{1 / p^{\prime}}\left\|(I+L)^{m+m_{1}} \varphi\right\|_{p, v} \\
& \leq\left\|\frac{1}{\left(1+\mu^{2}+\|\lambda\|^{2}\right)^{m_{1}}}\right\|_{1, \nu}(\nu(B(0, a)))^{1 / p^{\prime}} 2^{m+m_{1}} N_{p, m+m_{1}}(\varphi),
\end{aligned}
$$

which implies that

$$
\widetilde{P}_{m}(\varphi) \leq 2^{m+m_{1}}(\nu(B(0, a)))^{1 / p^{\prime}}\left\|\frac{1}{\left(1+\mu^{2}+\|\lambda\|^{2}\right)^{m_{1}}}\right\|_{1, \nu} N_{p, m+m_{1}}(\varphi),
$$

and the proof of the lemma is complete. 
Theorem 4.6. Let $a>0$ and $B^{\prime}$ a weakly* bounded set of $\mathscr{D}_{*, a}^{\prime}\left(\mathbb{R} \times \mathbb{R}^{n}\right)$. Then, there exists $m \in \mathbb{N}$ such that the elements of $B^{\prime}$ can be continuously extended to $\mathcal{W}_{a}^{m}\left(\mathbb{R} \times \mathbb{R}^{n}\right)$. Moreover, the family of these extensions is equicontinuous.

Proof. Let $p \in[1, \infty]$. Since $B^{\prime}$ is weakly* bounded in $D_{*, a}^{\prime}\left(\mathbb{R} \times \mathbb{R}^{n}\right)$, then from [14] and Lemma 4.5 there exist a positive constant $c$ and $m \in \mathbb{N}$ such that for all $T \in B^{\prime}$, for all $\varphi \in D_{*, a}\left(\mathbb{R} \times \mathbb{R}^{n}\right)$,

$$
|\langle T, \varphi\rangle| \leq c N_{p, m}(\varphi) .
$$

We consider the mappings

$$
\begin{gathered}
A: \mathscr{W}_{a}^{m}\left(\mathbb{R} \times \mathbb{R}^{n}\right) \longrightarrow\left(L^{p}(d \nu)\right)^{m+1}, \\
\varphi \longmapsto\left(L^{k} \varphi\right)_{0 \leq k \leq m},
\end{gathered}
$$

and for all $T \in B^{\prime}$,

$$
\begin{gathered}
L_{T}: A\left(D_{*, a}\left(\mathbb{R} \times \mathbb{R}^{n}\right)\right) \longrightarrow \mathbb{C}, \\
\left\langle L_{T}, A \varphi\right\rangle=\langle T, \varphi\rangle .
\end{gathered}
$$

From relation (4.51), we deduce that for all $\varphi \in D_{*, a}\left(\mathbb{R} \times \mathbb{R}^{n}\right)$,

$$
\left|\left\langle L_{T}, A \varphi\right\rangle\right| \leq c|| A \varphi \|_{\left(L^{p}(d v)\right)^{m+1}} .
$$

This means that $L_{T}$ is a continuous functional on the subspace $A\left(D_{*, a}\left(\mathbb{R} \times \mathbb{R}^{n}\right)\right)$ of the space $\left(L^{p}(d \nu)\right)^{m+1}$ and that for all $T \in B^{\prime}$,

$$
\left\|L_{T}\right\|_{A\left(D_{*, a}\left(\mathbb{R} \times \mathbb{R}^{n}\right)\right)}=\sup _{\|A \varphi\|_{\left(L^{p}(d v)\right)^{m+1}} \leq 1}\left|\left\langle L_{T}, A \varphi\right\rangle\right| \leq c .
$$

From the Hahn-Banach theorems, $L_{T}$ can be continuously extended on $\left(L^{p}(d \nu)\right)^{m+1}$, denoted again by $L_{T}$. Furthermore, for all $T \in B^{\prime}$,

$$
\left\|L_{T}\right\|_{\left(L^{p}(d v)\right)^{m+1}}=\sup _{\|\psi\|_{\left(L^{p}(d v)\right)^{m+1}} \leq 1}\left|\left\langle L_{T}, \psi\right\rangle\right|=\left\|L_{T}\right\|_{A\left(D_{*, a}\left(\mathbb{R} \times \mathbb{R}^{n}\right)\right)} \leq c .
$$

Now, from the Riez theorem, there exists $\left(f_{T, k}\right)_{0 \leq k \leq m} \subset L^{p^{\prime}}(d \nu)$ such that for all $\psi=$ $\left(\psi_{0}, \ldots, \psi_{m}\right) \in\left(L^{p}(d \nu)\right)^{m+1}$,

$$
\left\langle L_{T}, \psi\right\rangle=\sum_{k=0}^{m} \int_{\mathbb{R}^{n}} \int_{0}^{\infty} f_{T, k}(r, x) \psi_{k}(r, x) d \nu
$$

with

$$
\left\|L_{T}\right\|_{\left(L^{p}(d v)\right)^{m+1}}=\max _{0 \leq k \leq m}\left\|f_{T, k}\right\|_{p^{\prime}, v^{*}}
$$

Thus, from (4.56) it follows that for all $T \in B^{\prime}$, for all $k \in \mathbb{N}, 0 \leq k \leq m$,

$$
\left\|f_{T, k}\right\|_{p^{\prime}, v} \leq c
$$


In particular, for $\varphi \in W_{a}^{m}\left(\mathbb{R} \times \mathbb{R}^{n}\right)$ we have

$$
\left\langle L_{T}, A \varphi\right\rangle=\sum_{k=0}^{m} \int_{\mathbb{R}} \int_{0}^{\infty} f_{T, k}(r, x) L^{k}(\varphi)(r, x) d \nu(r, x) .
$$

Using Holder's inequality and relation (4.59), we get for all $T \in B^{\prime}$, for all $\varphi \in \mathcal{W}_{a}^{m}(\mathbb{R} \times$ $\left.\mathbb{R}^{n}\right)$,

$$
\left|\left\langle L_{T}, A \varphi\right\rangle\right| \leq(m+1) c[\nu(B(0, a))]^{1 / p} N_{\infty, m}(\varphi) .
$$

This shows that the mapping $L_{T} o A$ is a continuous extension of $T$ on $W_{a}^{m}\left(\mathbb{R} \times \mathbb{R}^{n}\right)$ and that the family $\left\{L_{T} o A\right\}_{T \in B^{\prime}}$ is equicontinuous, when applied to $\mathcal{W}_{a}^{m}\left(\mathbb{R} \times \mathbb{R}^{n}\right)$. This completes the proof of Theorem 4.6.

In the following, we will give a new characterization of the space $M_{p}^{\prime}\left(\mathbb{R} \times \mathbb{R}^{n}\right)$.

Theorem 4.7. Let $T \in S_{*}^{\prime}\left(\mathbb{R} \times \mathbb{R}^{n}\right), p \in\left[1, \infty\left[, p^{\prime}=p /(p-1)\right.\right.$. Then $T \in M_{p}^{\prime}\left(\mathbb{R} \times \mathbb{R}^{n}\right)$ if and only if for every $\varphi \in \mathscr{D}_{*}\left(\mathbb{R} \times \mathbb{R}^{n}\right)$, the function $T * \varphi$ belongs to the space $L^{p^{\prime}}(d \nu)$, where

$$
T * \varphi(r, x)=\left\langle T, \tau_{(r,-x)} \breve{\varphi}\right\rangle .
$$

Proof. Let $T \in M_{p}^{\prime}\left(\mathbb{R} \times \mathbb{R}^{n}\right)$. From Theorem 4.1, there exist $m \in \mathbb{N}$ and $f_{0}, \ldots, f_{m} \in L^{p^{\prime}}(d \nu)$ such that

$$
T=\sum_{k=0}^{m} L^{k} T_{f_{k}}
$$

in $\mathcal{M}_{p}^{\prime}\left(\mathbb{R} \times \mathbb{R}^{n}\right)$. Thus, for every $\varphi \in \mathscr{D}_{*}\left(\mathbb{R} \times \mathbb{R}^{n}\right)$,

$$
T * \varphi=\sum_{k=0}^{m} T_{f_{k}} * L^{k} \varphi=\sum_{k=0}^{m} f_{k} * L^{k} \varphi .
$$

Since, for all $k \in \mathbb{N}, 0 \leq k \leq m, f_{k} \in L^{p^{\prime}}(d \nu)$ and $L^{k} \varphi \in L^{1}(d \nu)$, then from inequality (2.24), we deduce that $f_{k} * L^{k} \varphi \in L^{p^{\prime}}(d \nu)$. This implies that the function $T * \varphi$ belongs to the space $L^{p^{\prime}}(d v)$.

Conversely, let $T \in S_{*}^{\prime}\left(\mathbb{R} \times \mathbb{R}^{n}\right)$ such that for every $\varphi \in \mathscr{D}_{*}\left(\mathbb{R} \times \mathbb{R}^{n}\right)$ the function $T * \varphi$ belongs to the space $L^{p^{\prime}}(d \nu)$. For $\varphi, \psi$ in $\mathscr{D}_{*}\left(\mathbb{R} \times \mathbb{R}^{n}\right)$, we have

$$
\left\langle T_{T * \varphi}, \psi\right\rangle=\langle T, \varphi * \breve{\psi}\rangle=\langle T, \psi * \breve{\varphi}\rangle=\left\langle T_{T * \psi}, \varphi\right\rangle .
$$

From Holder's inequality and using the hypothesis, we obtain

$$
\left|\left\langle T_{T * \varphi}, \psi\right\rangle\right| \leq\|T * \psi\|_{p^{\prime}, v}\|\varphi\|_{p, v},
$$


from which we deduce that the set

$$
B^{\prime}=\left\{T_{T * \varphi}, \varphi \in \mathscr{D}_{*}\left(\mathbb{R} \times \mathbb{R}^{n}\right) ;\|\varphi\|_{p, \nu} \leq 1\right\}
$$

is bounded in $\mathscr{D}_{*}^{\prime}\left(\mathbb{R} \times \mathbb{R}^{n}\right)$.

Now, using Theorem 4.6, it follows that for all $a>0$ there exists $m \in \mathbb{N}$ such that for all $\varphi \in \mathscr{D}_{*}\left(\mathbb{R} \times \mathbb{R}^{n}\right),\|\varphi\|_{p, v} \leq 1$, the mapping $T_{T * \varphi}$ can be continuously extended on the space $W_{a}^{m}\left(\mathbb{R} \times \mathbb{R}^{n}\right)$ and the family of these extensions is equicontinuous, which means that there exists $c>0$ such that for all $\varphi \in \mathscr{D}_{*}\left(\mathbb{R} \times \mathbb{R}^{n}\right),\|\varphi\|_{p, v} \leq 1$, for all $\psi \in \mathscr{W}_{a}^{m}(\mathbb{R} \times$ $\left.\mathbb{R}^{n}\right)$,

$$
\left|\left\langle T_{T * \varphi}, \psi\right\rangle\right| \leq c N_{\infty, m}(\psi)
$$

This involves that for all $\varphi \in \mathscr{D}_{*}\left(\mathbb{R} \times \mathbb{R}^{n}\right)$, for all $\psi \in \mathcal{W}_{a}^{m}\left(\mathbb{R} \times \mathbb{R}^{n}\right)$,

$$
\left|\left\langle T_{T * \varphi}, \psi\right\rangle\right| \leq c N_{\infty, m}(\psi)\|\varphi\|_{p, \nu} .
$$

On the other hand, we have for all $\varphi \in \mathscr{D}_{*}\left(\mathbb{R} \times \mathbb{R}^{n}\right)$, for all $\psi \in \mathcal{W}_{a}^{m}\left(\mathbb{R} \times \mathbb{R}^{n}\right)$,

$$
\left\langle T_{T * \varphi}, \psi\right\rangle=\left\langle T * T_{\psi}, \breve{\varphi}\right\rangle,
$$

where for all $\varphi \in S_{*}\left(\mathbb{R} \times \mathbb{R}^{n}\right)$,

$$
\left\langle T * T_{\psi}, \varphi\right\rangle=\left\langle T, T_{\psi} * \varphi\right\rangle=\langle T, \psi * \varphi\rangle .
$$

Relations (4.69) and (4.70) lead to for all $\varphi \in \mathscr{D}_{*}\left(\mathbb{R} \times \mathbb{R}^{n}\right)$,

$$
\left|\left\langle T * T_{\psi}, \varphi\right\rangle\right| \leq c N_{\infty, m}(\psi)\|\varphi\|_{p, \nu} .
$$

This last inequality shows that the functional $T * T_{\psi}$ can be continuously extended on the space $L^{p}(d v)$ and from Riez's theorem, there exists $g \in L^{p^{\prime}}(d v)$ such that

$$
T * T_{\psi}=T_{g} .
$$

Furthermore, from Proposition 4.4, there exist $s \in \mathbb{N}, \psi_{s} \in \mathcal{W}_{a}^{m}\left(\mathbb{R} \times \mathbb{R}^{n}\right)$, and $\varphi_{s} \in$ $\mathscr{D}_{*, a}\left(\mathbb{R} \times \mathbb{R}^{n}\right)$ satisfying

$$
\delta=(I+L)^{s} T_{\psi_{s}}+T_{\varphi_{s}},
$$

then

$$
T=(I+L)^{s}\left(T * T_{\psi_{s}}\right)+T * T_{\varphi_{s}}=(I+L)^{s}\left(T * T_{\psi_{s}}\right)+T_{T * \varphi_{s}} .
$$

We complete the proof by using the hypothesis, relation (4.73), and Theorem 4.1.

In the following, we will give a characterization of the bounded sets in $M_{p}^{\prime}\left(\mathbb{R} \times \mathbb{R}^{n}\right)$. 
Theorem 4.8. Let $p \in\left[1, \infty\left[\right.\right.$ and let $B^{\prime}$ be a subset of $M_{p}^{\prime}\left(\mathbb{R} \times \mathbb{R}^{n}\right)$. The following assertions are equivalent:

(i) $B^{\prime}$ is weakly bounded in $M_{p}^{\prime}\left(\mathbb{R} \times \mathbb{R}^{n}\right)$,

(ii) there exist $c>0$ and $m \in \mathbb{N}$ such that for every $T \in B^{\prime}$, it is possible to find $f_{0, T}, \ldots, f_{m, T} \subset L^{p^{\prime}}(d \nu)$ satisfying

$$
T=\sum_{k=0}^{m} L^{k} T_{f_{k}} \quad \text { with } \max _{0 \leq k \leq m}\left\|f_{k}\right\|_{p^{\prime}, v} \leq c,
$$

(iii) for every $\varphi \in \mathscr{D}_{*}\left(\mathbb{R} \times \mathbb{R}^{n}\right)$, the set $\{T * \varphi\}_{T \in B^{\prime}}$ is bounded in $L^{p^{\prime}}(d \nu)$.

Proof. (1) Suppose that $B^{\prime}$ is weakly* bounded in $M_{p}^{\prime}\left(\mathbb{R} \times \mathbb{R}^{n}\right)$, then from [14] $B^{\prime}$ is equicontinuous. There exist $c>0$ and $m \in \mathbb{N}$ such that

$$
\forall T \in B^{\prime}, \forall f \in \mathcal{M}_{p}\left(\mathbb{R} \times \mathbb{R}^{n}\right), \quad|\langle T, f\rangle| \leq c \gamma_{m, p}(f) .
$$

As in the proof of Theorem 4.6, we consider the mappings

$$
\begin{gathered}
A: \mathcal{M}_{p}\left(\mathbb{R} \times \mathbb{R}^{n}\right) \longrightarrow\left(L^{p}(d v)\right)^{m+1}, \\
f \longmapsto\left(f, g_{1}, \ldots, g_{m}\right)
\end{gathered}
$$

with

$$
L^{k} T_{f}=T_{g_{k}}, \quad 0 \leq k \leq m,
$$

and for all $T \in B^{\prime}$,

$$
\begin{gathered}
L_{T}: A\left(M_{p}\left(\mathbb{R} \times \mathbb{R}^{n}\right)\right) \longrightarrow \mathbb{C}, \\
\left\langle L_{T}, A(f)\right\rangle=\langle T, f\rangle .
\end{gathered}
$$

Then, relation (4.77) implies that for all $\varphi \in \mathcal{M}_{p}\left(\mathbb{R} \times \mathbb{R}^{n}\right)$,

$$
\left|L_{T}(A \varphi)\right| \leq c\|A \varphi\|_{\left(L^{p}(d v)\right)^{m+1}} .
$$

Using Hahn-Banach's theorem and Riez's theorem, we deduce that $L_{T}$ can be continuously extended on $\left(L^{p}(d \nu)\right)^{m+1}$, denoted again by $L_{T}$, and that there exists $\left(f_{T, k}\right)_{0 \leq k \leq m} \subset$ $L^{p^{\prime}}(d \nu)$ verifying for all $\psi=\left(\psi_{0}, \ldots, \psi_{m}\right) \in\left(L^{p}(d \nu)\right)^{m+1}$,

$$
\left\langle L_{T}, \psi\right\rangle=\sum_{k=0}^{m} \int_{\mathbb{R}^{n}} \int_{0}^{\infty} f_{T, k}(r, x) \psi_{k}(r, x) d \nu(r, x)
$$

with

$$
\left\|L_{T}\right\|_{\left(L^{p}(d v)\right)^{m+1}}=\max _{0 \leq k \leq m}\left\|f_{T, k}\right\|_{p^{\prime}, \nu} \leq c .
$$


In particular, if $\psi=A(f), f \in M_{p}\left(\mathbb{R} \times \mathbb{R}^{n}\right)$,

$$
\left\langle L_{T}, A(f)\right\rangle=\langle T, f\rangle=\sum_{k=0}^{m}\left\langle L^{k} T_{f_{T, k}}, f\right\rangle .
$$

This proves that $(\mathrm{i}) \Rightarrow$ (ii).

(2) Suppose that there exist $c>0$ and $m \in \mathbb{N}$ such that for every $T \in B^{\prime}$ we can find $f_{0, T}, \ldots, f_{m, T} \subset L^{p^{\prime}}(d \nu)$ satisfying

$$
T=\sum_{k=0}^{m} L^{k} T_{f_{T, k}}, \quad \max _{0 \leq k \leq m}\left\|f_{T, k}\right\|_{p^{\prime}, v} \leq c .
$$

Then for all $f \in \mathcal{M}_{p}\left(\mathbb{R} \times \mathbb{R}^{n}\right)$, for all $T \in B^{\prime}$,

$$
\langle T, f\rangle=\sum_{k=0}^{m} \int_{\mathbb{R}^{n}} \int_{0}^{\infty} f_{T, k}(r, x) g_{k}(r, x) d \nu(r, x),
$$

consequently, for all $T \in B^{\prime}$, for all $f \in \mathcal{M}_{p}\left(\mathbb{R} \times \mathbb{R}^{n}\right)$,

$$
|\langle T, f\rangle| \leq(m+1) c \gamma_{m, p}(f),
$$

which means that the set $B^{\prime}$ is weakly* bounded in $M_{p}^{\prime}\left(\mathbb{R} \times \mathbb{R}^{n}\right)$ and proves that (ii) $\Rightarrow(\mathrm{i})$.

(3) Suppose that (ii) holds. Let $\varphi \in \mathscr{D}_{*}\left(\mathbb{R} \times \mathbb{R}^{n}\right)$, then from Theorem 4.7 we know that for all $T \in B^{\prime}$, the function $T * \varphi$ belongs to the space $L^{p^{\prime}}(d \nu)$. But

$$
T * \varphi=\sum_{k=0}^{m} T_{f_{k}} * L^{k} \varphi
$$

consequently, for all $T \in B^{\prime}$,

$$
\|T * \varphi\|_{p^{\prime}, \nu} \leq(m+1) c \gamma_{m, p}(\varphi)
$$

This shows that the set $\{T * \varphi\}_{T \in B^{\prime}}$ is bounded in $L^{p^{\prime}}(d \nu)$ and therefore (ii) involves (iii).

(4) Suppose that (iii) holds. Let $T \in B^{\prime}$; for all $\varphi, \psi \in \mathscr{D}_{*}\left(\mathbb{R} \times \mathbb{R}^{n}\right)$, we have

$$
\left|\left\langle T_{T * \varphi}, \psi\right\rangle\right|=\left|\left\langle T_{T * \psi}, \varphi\right\rangle\right| \leq\|T * \psi\|_{p^{\prime}, \nu}\|\varphi\|_{p, \nu},
$$

from which we deduce that the set

$$
B^{\prime}=\left\{T_{T * \varphi}, T \in B^{\prime}, \varphi \in \mathscr{D}_{*}\left(\mathbb{R} \times \mathbb{R}^{n}\right) ;\|\varphi\|_{p, \nu} \leq 1\right\}
$$

is bounded in $\mathscr{D}_{*}^{\prime}\left(\mathbb{R} \times \mathbb{R}^{n}\right)$.

Now, using Theorem 4.6, it follows that for all $a>0$, there exists $m \in \mathbb{N}$ such that for all $\varphi \in \mathscr{D}_{*}\left(\mathbb{R} \times \mathbb{R}^{n}\right),\|\varphi\|_{p, v} \leq 1$, and $T \in B^{\prime}$, the mapping $T_{T * \varphi}$ can be continuously extended on the space $W_{a}^{m}\left(\mathbb{R} \times \mathbb{R}^{n}\right)$ and the family of these extensions is equicontinuous, 
which means that there exists $c>0$ satisfying for all $T \in B^{\prime}$, for all $\varphi \in \mathscr{D}_{*}\left(\mathbb{R} \times \mathbb{R}^{n}\right)$; for all $\psi \in W_{a}^{m}\left(\mathbb{R} \times \mathbb{R}^{n}\right)$, (4.69) holds. On the other hand, for every $T \in B^{\prime}$, we have for all $\varphi \in \mathscr{D}_{*}\left(\mathbb{R} \times \mathbb{R}^{n}\right)$, for all $\psi \in \mathscr{W}_{a}^{m}\left(\mathbb{R} \times \mathbb{R}^{n}\right),(4.70)$ holds. From relations (4.69) and (4.70), we deduce that the functional $T * T_{\psi}$ can be continuously extended on the space $L^{p}(d \nu)$ and from Riez's theorem, there exist $g_{T, \psi} \in L^{p^{\prime}}(d \nu)$ such that

$$
T * T_{\psi}=T_{g_{T, \psi}}
$$

However, relations (4.69) and (4.70) involve that for all $T \in B^{\prime}$,

$$
\left\|g_{T, \psi}\right\|_{p^{\prime}, v} \leq c N_{\infty, m}(\psi)
$$

Again by Proposition 4.4, it follows that there exist $s \in \mathbb{N}, \psi_{s} \in \mathcal{W}_{a}^{m}\left(\mathbb{R} \times \mathbb{R}^{n}\right)$, and $\varphi_{s} \in$ $\mathscr{D}_{*, a}\left(\mathbb{R} \times \mathbb{R}^{n}\right)$ verifying for all $T \in B^{\prime}$,

$$
T=T * \delta=(I+L)^{s}\left(T * T_{\psi_{s}}\right)+T_{T * \varphi_{s}}
$$

and by relation (4.92) we get

$$
T=(I+L)^{s} T_{g_{T, s}}+T_{T * \varphi_{s}}
$$

Thus, from the hypothesis we obtain,

$$
\forall T \in B^{\prime}, \quad\left\|T * \varphi_{s}\right\|_{p^{\prime}, v} \leq c_{s}
$$

and using relation (4.93), we have

$$
\forall T \in B^{\prime}, \quad\left\|g_{T, s}\right\|_{p^{\prime}, v} \leq c N_{\infty, m}\left(\varphi_{s}\right)
$$

This completes the proof.

\section{Convolution product on the space $M_{p}^{\prime}\left(\mathbb{R} \times \mathbb{R}^{n}\right) \times M_{r}\left(\mathbb{R} \times \mathbb{R}^{n}\right)$}

In this section, we define and study a convolution product on the space $M_{p}^{\prime}\left(\mathbb{R} \times \mathbb{R}^{n}\right) \times$ $M_{r}\left(\mathbb{R} \times \mathbb{R}^{n}\right), 1 \leq r \leq p<\infty$, where $M_{r}\left(\mathbb{R} \times \mathbb{R}^{n}\right)$ is the closure of the space $S_{*}\left(\mathbb{R} \times \mathbb{R}^{n}\right)$ in $M_{r}\left(\mathbb{R} \times \mathbb{R}^{n}\right)$.

Proposition 5.1. Let $p \in\left[1, \infty\left[\right.\right.$. For every $(r, x) \in\left[0, \infty\left[\times \mathbb{R}^{n}\right.\right.$, the operator $\tau_{(r, x)}$ given by Definition 2.2(i), is a continuous mapping from $\mu_{p}\left(\mathbb{R} \times \mathbb{R}^{n}\right)$ into itself.

Proof. Let $f \in \mathcal{M}_{p}\left(\mathbb{R} \times \mathbb{R}^{n}\right)$ and $g_{k} \in L^{p}(d v)$ such that

$$
T_{g_{k}}=L^{k} T_{f}, \quad k \in \mathbb{N} .
$$


Then for all $\varphi \in S_{*}\left(\mathbb{R} \times \mathbb{R}^{n}\right)$,

$$
\left\langle L^{k} T_{\tau_{(r, x)} f}, \varphi\right\rangle=\left\langle T_{\tau_{(r,-x)}} \breve{g}_{k}, \varphi\right\rangle
$$

Since the operator $\tau_{(r, x)}$ is continuous from $L^{p}(d \nu)$ into itself, we deduce that for all $f \in$ $\mu_{p}\left(\mathbb{R} \times \mathbb{R}^{n}\right)$ and $(r, x) \in\left[0, \infty\left[\times \mathbb{R}^{n}\right.\right.$, the function $\tau_{(r, x)} f$ belongs to the space $\mathcal{M}_{p}(\mathbb{R} \times$ $\left.\mathbb{R}^{n}\right)$. Moreover,

$$
\gamma_{m, p}\left(\tau_{(r, x)} f\right)=\max _{0 \leq k \leq m}\left\|\tau_{(r,-x)} \breve{g_{k}}\right\|_{p, v} \leq \max _{0 \leq k \leq m}\left\|g_{k}\right\|_{p, v}=\gamma_{m, p}(f)
$$

which shows that the operator $\tau_{(r, x)}$ is continuous from $\mu_{p}\left(\mathbb{R} \times \mathbb{R}^{n}\right)$ into itself.

Definition 5.2. A convolution product of $T \in M_{p}^{\prime}\left(\mathbb{R} \times \mathbb{R}^{n}\right)$ and $f \in M_{p}\left(\mathbb{R} \times \mathbb{R}^{n}\right)$ is defined by for all $(r, x) \in\left[0, \infty\left[\times \mathbb{R}^{n}\right.\right.$,

$$
T * f(r, x)=\left\langle T, \tau_{(r,-x)} \breve{f}\right\rangle .
$$

Let $T \in M_{p}^{\prime}\left(\mathbb{R} \times \mathbb{R}^{n}\right) ; T=\sum_{k=0}^{m} L^{k} T_{f_{k}}$ with $\left\{f_{k}\right\}_{0 \leq k \leq m} \subset L^{p^{\prime}}(d \nu)$ and $\phi \in M_{r}\left(\mathbb{R} \times \mathbb{R}^{n}\right)$, $1 \leq r \leq p$, then for all $k \in \mathbb{N}$, there exists $\phi_{k} \in L^{r}(d \nu)$ such that $T_{\phi_{k}}=L^{k} T_{\phi}$. From inequality (2.24), it follows that for $0 \leq k \leq m$, the function $f_{k} * \phi_{k}$ belongs to the space $L^{q}(d \nu)$ with $1 / q=1 / r+1 / p^{\prime}-1=1 / r-1 / p$ and by using the density of $S_{*}\left(\mathbb{R} \times \mathbb{R}^{n}\right)$ in $M_{r}\left(\mathbb{R} \times \mathbb{R}^{n}\right)$, we deduce that the expression $\sum_{k=0}^{m} f_{k} * \phi_{k}$ is independent of the sequence $\left\{f_{k}\right\}_{0 \leq k \leq m}$. Then, we put

$$
T * \phi=\sum_{k=0}^{m} f_{k} * \phi_{k} .
$$

This allows us to say that

$$
M_{p}^{\prime}\left(\mathbb{R} \times \mathbb{R}^{n}\right) * M_{r}\left(\mathbb{R} \times \mathbb{R}^{n}\right) \subset L^{q}(d v) .
$$

Lemma 5.3. Let $1 \leq r \leq p<\infty, T \in M_{p}^{\prime}\left(\mathbb{R} \times \mathbb{R}^{n}\right)$, and $\phi \in M_{r}\left(\mathbb{R} \times \mathbb{R}^{n}\right)$. Then, for all $k \in \mathbb{N}$

$$
L^{k} T_{T * \phi}=T_{T * \phi_{k}}
$$

with $T_{\phi_{k}}=L^{k} T_{\phi}$.

Proof. If $\phi \in S_{*}\left(\mathbb{R} \times \mathbb{R}^{n}\right)$, then the function $T * \phi$ is infinitely differentiable and we have

$$
L^{k}\left(T_{T * \phi}\right)=T_{L^{k}(T * \phi)}=T_{T * L^{k} \phi}
$$

Therefore, the result follows from the density of $S_{*}\left(\mathbb{R} \times \mathbb{R}^{n}\right)$ in $M_{r}\left(\mathbb{R} \times \mathbb{R}^{n}\right)$.

Proposition 5.4. Let $1 \leq r \leq p<\infty$ and $q \in[1, \infty]$ such that

$$
\frac{1}{q}=\frac{1}{r}-\frac{1}{p}
$$


Then for every $T \in M_{p}^{\prime}\left(\mathbb{R} \times \mathbb{R}^{n}\right)$, the mapping

$$
\phi \longrightarrow T * \phi
$$

is continuous from $M_{r}\left(\mathbb{R} \times \mathbb{R}^{n}\right)$ into $M_{q}\left(\mathbb{R} \times \mathbb{R}^{n}\right)$.

Proof. Let $T \in \mathcal{M}_{p}^{\prime}\left(\mathbb{R} \times \mathbb{R}^{n}\right) ; T=\sum_{k=0}^{m} L^{k} T_{f_{k}}$ with $\left\{f_{k}\right\}_{0 \leq k \leq m} \subset L^{p^{\prime}}(d \nu)$, then for $\phi \in$ $M_{r}\left(\mathbb{R} \times \mathbb{R}^{n}\right), 1 \leq r \leq p$, and by using relation (5.5), we get $T * \phi=\sum_{k=0}^{m} f_{k} * \phi_{k}$, where $\phi_{k} \in L^{r}(d \nu)$ and

$$
T_{\phi_{k}}=L^{k} T_{\phi}
$$

From Lemma 5.3, we have for all $s \in \mathbb{N}$, for all $\phi \in M_{r}\left(\mathbb{R} \times \mathbb{R}^{n}\right)$,

$$
L^{s} T_{T * \phi}=T_{T * \phi_{s}}
$$

Using relation (5.6), we deduce that the function $T * \phi$ belongs to the space $M_{q}\left(\mathbb{R} \times \mathbb{R}^{n}\right)$.

On the other hand, from relation (5.12), we obtain

$$
\gamma_{l, q}(T * \phi)=\max _{0 \leq s \leq l}\left\|T * \phi_{s}\right\|_{q, v}
$$

According to relation (5.12), we have

$$
T * \phi_{s}=\sum_{k=0}^{m} f_{k} * \phi_{k+s}
$$

consequently,

$$
\left\|T * \phi_{s}\right\|_{q, \nu} \leq \sum_{k=0}^{m}\left\|f_{k}\right\|_{p^{\prime}, v}\left\|\phi_{k+s}\right\|_{r, v} \leq\left(\sum_{k=0}^{m}\left\|f_{k}\right\|_{p^{\prime}, v}\right) \gamma_{m+l, r}(\phi) .
$$

Hence

$$
\gamma_{l, q}(T * \phi) \leq\left(\sum_{k=0}^{m}\left\|f_{k}\right\|_{p^{\prime}, v}\right) \gamma_{m+l, r}(\phi)
$$

which proves the result.

Definition 5.5. Let $1 \leq p, q, r<\infty$ such that (5.9) holds. A convolution product of $T \in$ $\mathcal{M}_{p}^{\prime}\left(\mathbb{R} \times \mathbb{R}^{n}\right)$ and $S \in \mathcal{M}_{q}^{\prime}\left(\mathbb{R} \times \mathbb{R}^{n}\right)$ is defined by for all $\phi \in M_{r}\left(\mathbb{R} \times \mathbb{R}^{n}\right)$,

$$
\langle S * T, \phi\rangle=\langle S, T * \phi\rangle .
$$

From this definition and Proposition 5.4 we deduce the following result.

Proposition 5.6. Let $1 \leq p, q, r<f \infty$ such that (5.9) holds. Then, for all $T \in M_{p}^{\prime}\left(\mathbb{R} \times \mathbb{R}^{n}\right)$ and $S \in M_{q}^{\prime}\left(\mathbb{R} \times \mathbb{R}^{n}\right)$, the functional $S * T$ is continuous on $M_{r}\left(\mathbb{R} \times \mathbb{R}^{n}\right)$. 


\section{References}

[1] S. Abdullah, On convolution operators and multipliers of distributions of $L^{p}$-growth, J. Math. Anal. Appl. 183 (1994), no. 1, 196-207.

[2] S. Abdullah and S. Pilipović, Bounded subsets in spaces of distributions of $L^{p}$-growth, Hokkaido Math. J. 23 (1994), no. 1, 51-54.

[3] L. E. Andersson, On the determination of a function from spherical averages, SIAM J. Math. Anal. 19 (1988), no. 1, 214-232.

[4] J. Barros-Neto, An Introduction to the Theory of Distributions, Pure and Applied Mathematics, vol. 14, Marcel Dekker, New York, 1973.

[5] J. J. Betancor and B. J. González, Spaces of $\mathscr{D}_{L^{p}}$-type and the Hankel convolution, Proc. Amer. Math. Soc. 129 (2001), no. 1, 219-228.

[6] J. A. Fawcett, Inversion of n-dimensional spherical averages, SIAM J. Appl. Math. 45 (1985), no. 2, 336-341.

[7] H. Hellsten and L. E. Andersson, An inverse method for the processing of synthetic aperture radar data, Inverse Problems 3 (1987), no. 1, 111-124.

[8] M. Herberthson, A numerical implementation of an inversion formulas for CARABAS raw data, Internal Report D 30430-3.2, National Defense Research Institute, FOA, Box 1165; S-581 11, Linköping, Sweden, 1986.

[9] N. N. Lebedev, Special Functions and Their Applications, Dover Publications, New York, 1972.

[10] M. M. Nessibi, L. T. Rachdi, and K. Trimèche, Ranges and inversion formulas for spherical mean operator and its dual, J. Math. Anal. Appl. 196 (1995), no. 3, 861-884.

[11] L. T. Rachdi and K. Trimèche, Weyl transforms associated with the spherical mean operator, Analysis and Applications 1 (2003), no. 2, 141-164.

[12] L. Schwartz, Theory of Distributions. I, Hermann, Paris, 1957.

[13] _ Theory of Distributions. II, Hermann, Paris, 1959.

[14] F. Trèves, Topological Vector Spaces, Distributions and Kernels, Academic Press, New York, 1967.

[15] G. N. Watson, A Treatise on the Theory of Bessel Functions, 2nd ed., Cambridge University Press, Cambridge, 1966.

M. Dziri: Department of Mathematics, Faculty of Sciences of Tunis, University of Tunis, 1060 Tunis, Tunisia

E-mail address: moncef.dziri@iscae.rnu.tn

M. Jelassi: Department of Mathematics, Faculty of Sciences of Tunis, University of Tunis, 1060 Tunis, Tunisia

E-mail address: mouradjelassi@fst.rnu.tn

L. T. Rachdi: Department of Mathematics, Faculty of Sciences of Tunis, University of Tunis, 1060 Tunis, Tunisia

E-mail address: lakhdartannech.rachdi@fst.rnu.tn 


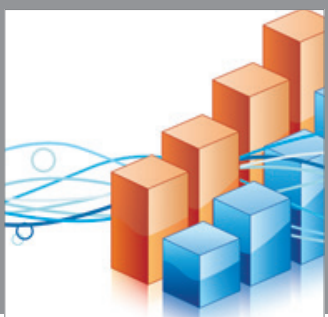

Advances in

Operations Research

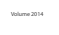

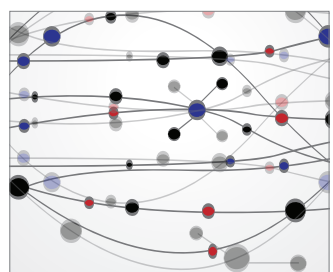

\section{The Scientific} World Journal
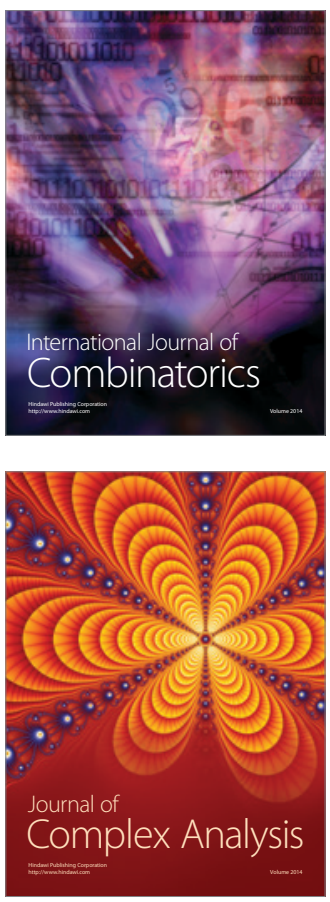

International Journal of

Mathematics and

Mathematical

Sciences
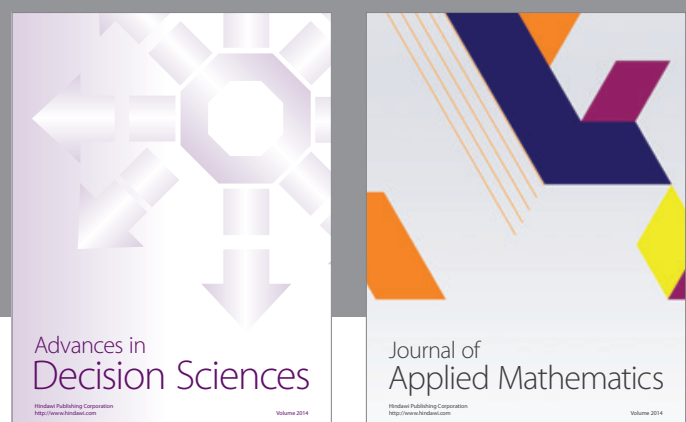

Journal of

Applied Mathematics
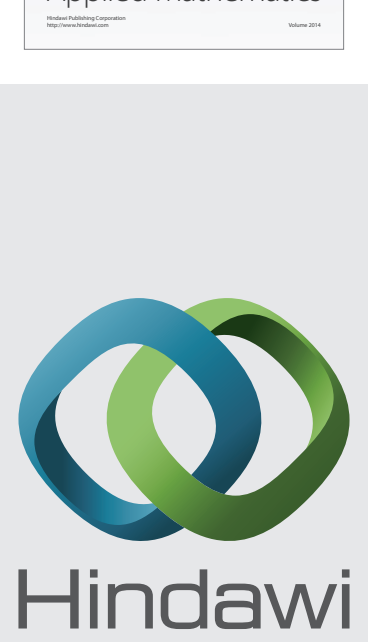

Submit your manuscripts at http://www.hindawi.com
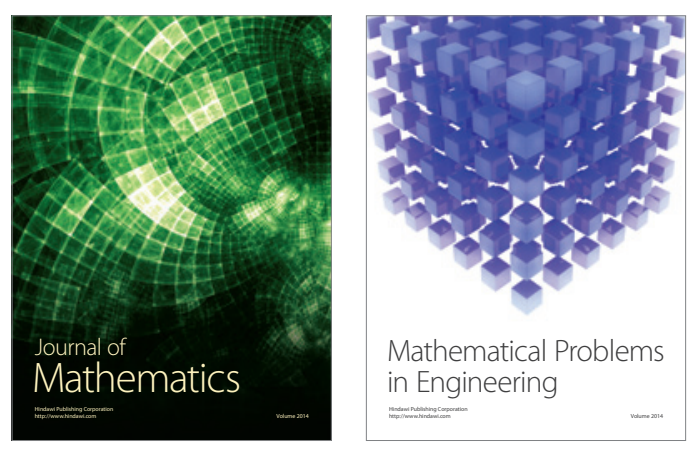

Mathematical Problems in Engineering
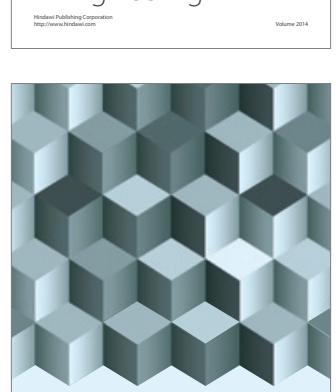

Journal of

Function Spaces
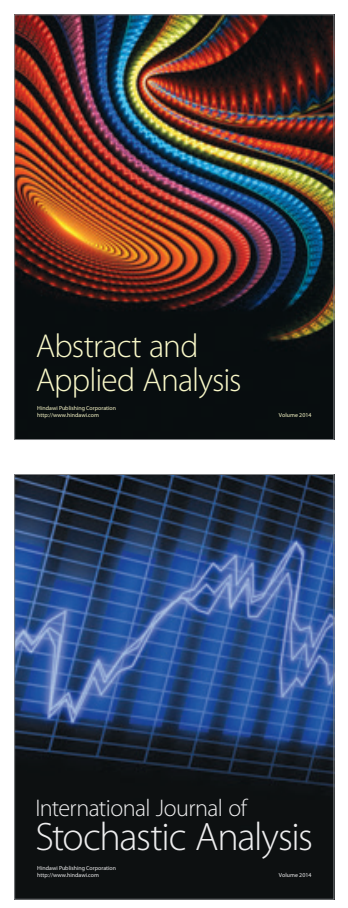

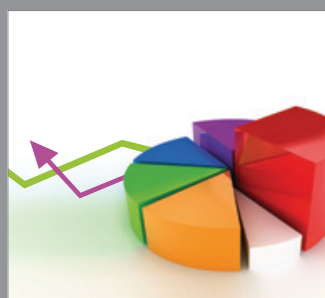

ournal of

Probability and Statistics

Promensencen
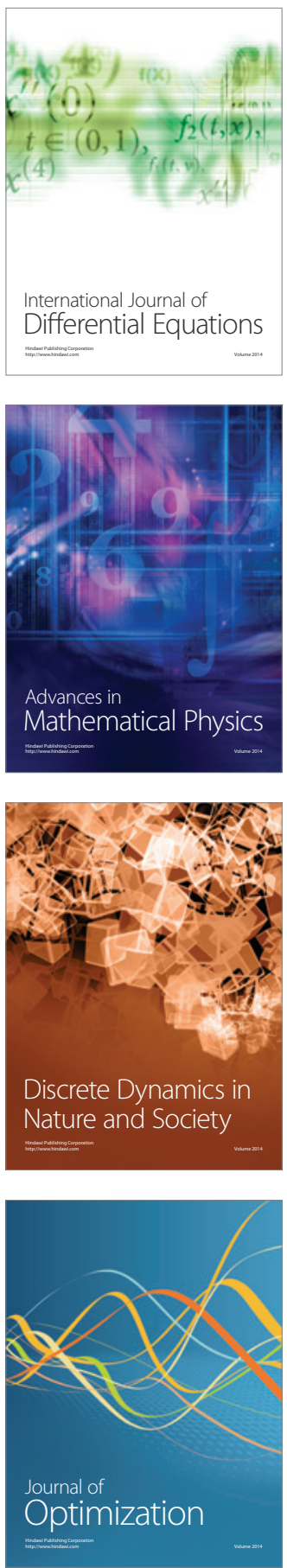